\title{
Aspects of automation in a lobster farming plant
}

\author{
STEINAR GRIMSEN $\dagger$, ROBERT N. JAQUES $\ddagger$, \\ VINCENT ERENST $\dagger$ and JENS G. BALCHEN§
}

Keywords: Aquaculture, lobster farming, automation, instrumentation, computer control.

This paper describes the automation aspects of rearing juvenile lobsters for sea ranching. It gives an outline of rearing techniques and presents specifications for a feeding system. A description is given of the equipment that has been developed. Other automation and instrumentation tasks are briefly discussed.

\section{Introduction}

The American lobster (Homarus americanus) and the European lobster ( $H$. gammarus) both support a valuable fishery, although the stocks of the American lobster are far greater than its European counterpart. Landings in 1982 were 40683 tonnes, and 1877 tonnes for respectively U.S.A./Canada and Europe (FAO Yearbook of Fishery Statistics 1982). However on both sides of the Atlantic the stocks show signs of overexploitation with reduced catches in traditional fishery areas and reduced catch per unit of effort. Norway in particular has had an alarming reduction in landings from about 1000 tonnes per year in the 1930's to 73 tonnes in 1982 , and the downward trend is being maintained.

With reduction in availability and the concomitant rise in prices the lobster has become an attractive candidate for aquaculture both in North America and Europe. However up to the present time growth of lobsters from egg to market size under controlled conditions on land has not been proved to be commercially viable (Wickins, 1983; Beard et al., 1985).

There is however an alternative approach to farming lobsters which involves growing juveniles up to a certain size on land and afterwards releasing them into the sea, where growth to market size would take place. Depending on the situation this can be seen as stock enhancement, or under a more controlled form, as sea ranching.

Production and release of hatchery reared lobsters to augment the fishery is by no means an innovation. Between 1885 and 1920 more than 22 lobster hatcheries were established in the USA, Canada and Europe, including Norway (Carlson, 1954). However it was not possible to determine to what extent these lobsters, mostly released as postlarvae, contributed to the natural population and fishery catch. As a result most of the restocking programmes were discontinued.

Received 15 January 1987.

This paper was presented at the IFAC Symposium on Automation and Dataprocessing in Aquaculture, Trondheim, Norway, 18-20 August 1986.

This paper is reprinted with the permission of IFAC.

$\uparrow$ Tiedemanns Lobster Project, P.O. Box 131, N 7201 Kyrksæterøra (Norway).

$\ddagger$ Aqua Unique A.S., P.O. Box 9132, Vaterland, N 0134 Oslo-1 (Norway).

$\S$ Division of Engineering Cybernetics, The Norwegian Institute of Technology, University of Trondheim, N 7034 Trondheim-NTH (Norway). 
In 1978 the idea of releasing hatchery reared lobsters into the sea was revived with a cooperation project between SINTEF (Foundation for Scientific and Industrial Research at the Norwegian Institute of Technology) and a Norwegian industrial company Tiedemanns. The concept of this project was different in that it involved growing the lobsters under optimal temperature conditions for up to one year on land before releasing them into the sea in limited areas with artificial or natural barriers. After growing to market size in the sea, the lobsters would then be harvested. The results of this lobster ranching will be known in 1988-1989, when 100000 juveniles released at a selected site on the west coast of Norway will be ready for harvesting.

In 1980, after successful experimentation with both the land and sea aspects of lobster farming, Tiedemanns decided to build a large scale production unit. This was built at Holla Smelting Works, Kyrksæterøra in mid Norway, where there is an abundant supply of heated sea water available. The annual production capacity of this unit is at present 120000 year old juveniles.

Such a large scale operation requires new technological solutions for the efficient running of the plant. Automation is essential for some aspects, e.g. feeding, and desirable for many others. This paper describes technology already developed and applied, and discusses perspectives for future developments.

\section{Lobster farming principles}

The techniques for producing juvenile lobsters at the Tiedemanns plant are in principle the same as those described in the literature (Van Olst et al., 1980; Chang and Conklin, 1983; Beard et al., 1985). Production includes: (1) communal rearing to post-larva stage, and (2) ongrowing of separately housed postlarvae or juveniles. The procedures have been modified to meet the requirements of a large scale plant. A brief outline follows.

Berried females $(0.8-2 \mathrm{~kg})$, brought from fishermen in early summer, are selected into groups, according to the state of development of their eggs. Females with highly developed eggs are placed in individual boxes with flowing sea water (1-2 $1 / \min ; 18-20^{\circ} \mathrm{C}$ ). At hatching, the free swimming larvae are taken by the waterflow and collect in a net-screened box positioned at the water outlet. 6000-7000 larvae are stocked in a 2501 fibreglass bin. Here larvae and feed are continuously stirred in a spiral upwelling pattern, generated by the incoming water $\left(8-10 \mathrm{l} / \mathrm{min} ; 20-22^{\circ} \mathrm{C}\right)$. Good dispersion of the larvae reduces cannibalism. The larvae are fed with live or frozen brine shrimp (Artemia salina) 2-3 times per day. Brine shrimp are cultured at the plant using a system described by Brisset et al. (1982). After a period of 12-18 days the lobster larvae develop into postlarvae.

These postlarvae are manually transferred to separate boxes $(13.5 \times 9.0$ $\times 12.5 \mathrm{~cm}$ ) in a large circular pool (Fig. 1). The pool is divided in 11 concentric rings (width $180 \mathrm{~cm}$, waterdepth $25 \mathrm{~cm}$ ). The lobster boxes grouped in units of 120 , circulate in the rings driven by lines of water jets. All the feeding, sorting and inspection work can be done from a bridge that runs from the centre of the pool to the outermost ring.

The juveniles are fed 5 times a week with brine shrimp or a moist pellet (feed ration $5-10 \%$ of lobster wet weight per feeding), distributed by an automatic feeding system. At a water temperature of $20^{\circ} \mathrm{C}$ a size of 8-10 gram has been reached in 11-12 months. 


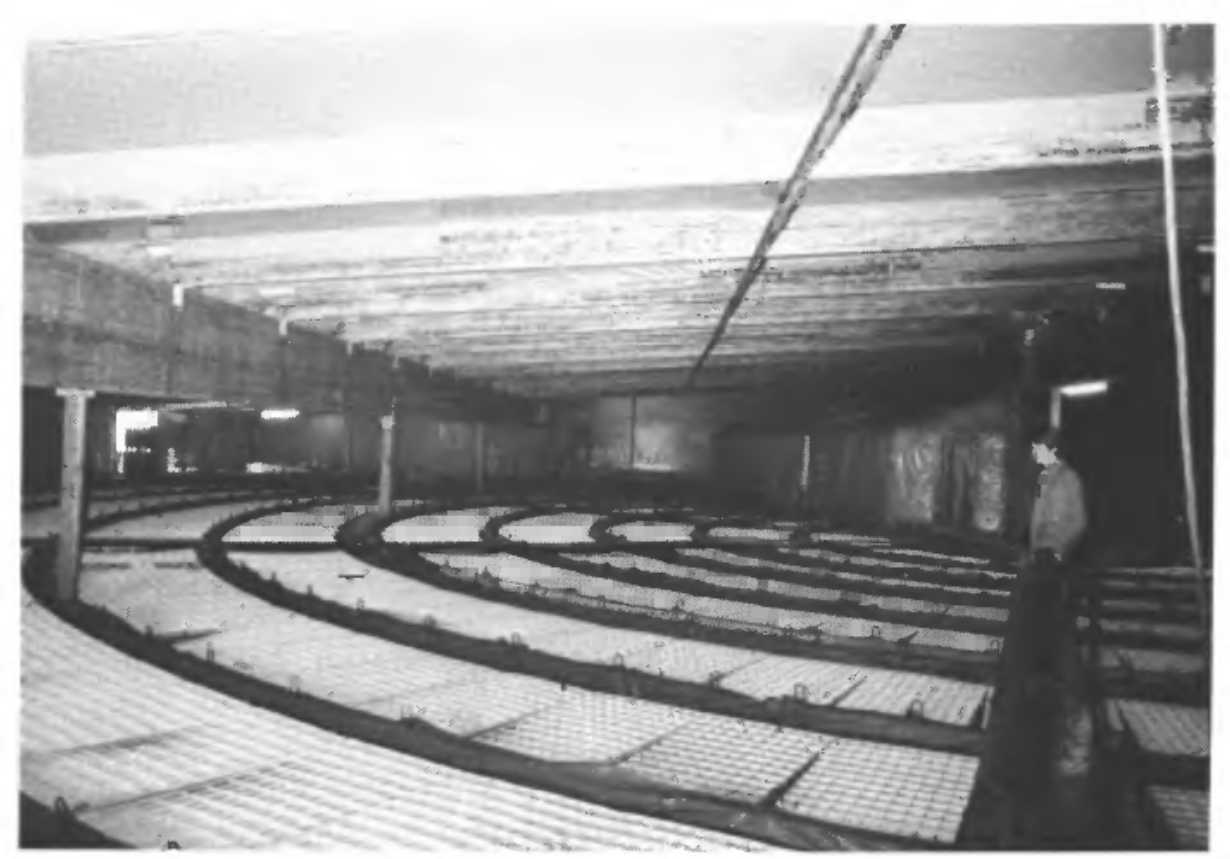

Figure 1. Circular pool.

The juveniles are transported to the release sites packed in cardboard boxes with moist wood chips. At a temperature of $8-10^{\circ} \mathrm{C}$ and a duration of transport less than 24 hours, survival is $98-100 \%$. Suitable sites are selected using criteria concerning depth, substrate and abundance of feed organisms for lobsters. Release density presently used is one juvenile per $10-20 \mathrm{~m}^{2}$.

\section{Importance of automation}

Intensive culture of lobsters requires, because of the cannibalistic nature of the animals, separate housing of each individual. Apart from a complicated lay out of a plant, this entails labour intensive husbandry.

In the production scheme outlined above individual treatment of the juveniles is desirable in a number of work routines. If these were to be performed manually, production of 120000 juveniles per year would require 6-7 workers, and the cost of the operation would be prohibitively high. Furthermore the performance of repetitive tasks often leads to reduced worker effectiveness with a consequent negative influence on the production process. Automation is thus a necessity, reducing labour costs and improving the quality of the product.

\section{Prototype of a computer controlled feeding system}

When designing the plant at Kyrksæterøra, it was estimated that mortality in the second production phase would be $25-30 \%$. To fill up the boxes in which a lobster had died, new postlarvae were to be produced several times a year. Every unit of 120 boxes would then contain several sizes of lobsters. To obtain optimal growth and avoid accumulation or food waste every lobster should receive a feed portion in accordance with its size. With these things in mind, a feeding system was developed that administered the right portion to every lobster and only to boxes containing a live lobster. 


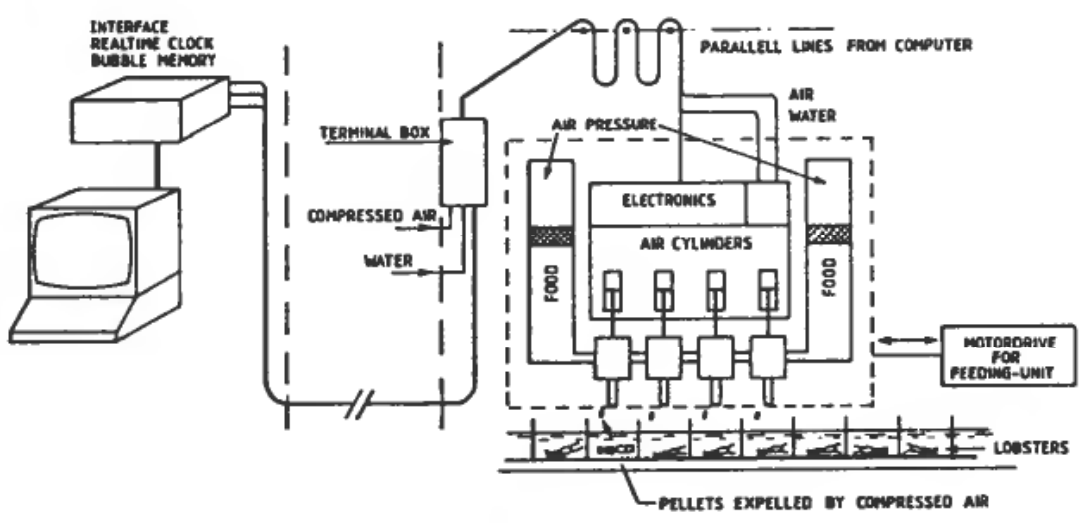

Figure 2. Schematic of prototype equipment.

The prototype equipment consisted of a feeding machine controlled by an Apple II computer with a $120 \mathrm{~K}$-byte magnetic bubble memory and a real time clock as illustrated in Fig. 2.

Every single box had one byte in the magnetic bubble memory. This byte showed if the box contained a lobster, the age of the lobster and the number of times the box had been refilled. Registration of dead/alive and refilling had to be done manually, but it was planned that in time a T.V. camera and image processing would be developed. At filling or refilling a box the age would be set to zero. The real time clock would update the age every fourteenth day. A feeding table coupling age and amount of feed was part of the computer program.

The feeding machine moves over the bridge driven by an electromotor. For every ring there were three feeding stops indicated by marks on the bridge. The moist feed was transported from a 501 container to four identical feeding cylinders with the aid of compressed air. Every feeding cylinder fed one lobster when moved in and out. The amount of feed depended on the length of the pellet produced by the feeding cylinder. Each cylinder could produce 32 pellet sizes independent of the others.

Every unit of 120 boxes could be identified by optical reading of a plastic card mounted on the top of the unit. These cards had 12 tongues of which the 10 central ones could be removed to form a binary code. A detector mounted on the feeding machine counted the individual boxes to determine the position of the feeder in the box unit.

\section{Experiences and modifications}

When production of lobsters was started in the new plant, it appeared that mortality in the second production phase was only $10-15 \%$. In addition, with the compound moist feed that was developed, it was shown that overfeeding caused no problems. Within certain limits the lobsters would always chew the feed into small pieces that would be removed by the incoming water.

The prototype equipment did not perform as well as hoped. Data between the computer and the feeder were conveyed over parallel lines and the chosen components were very sensitive to electrical noise. Atmospheric humidity caused a lot of contact problems.

It was decided that individual treatment of every lobster was not a necessity. 


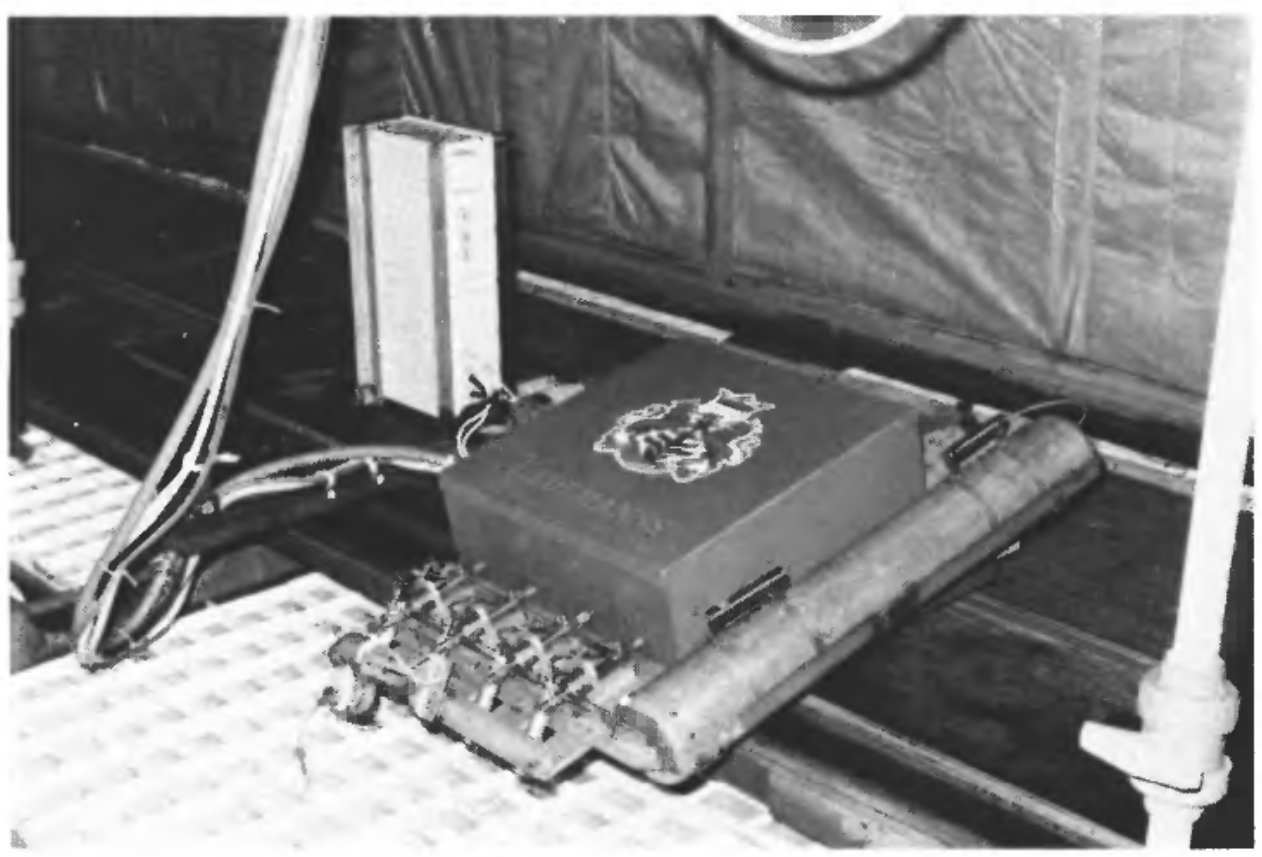

Figure 3. The feeding equipment.

With some manual sorting the size variation of the lobsters in one ring could be held within acceptable limits. Average size could then be used to determine the feeding level.

The feeding equipment was simplified in order to increase speed and the reliability. The use of the concept one lobster-one byte was discontinued. Pellet size is now regulated only for each ring i.e. all the lobsters in one ring receive the same pellet size. The mechanical part of the system is basically the same as in the prototype (Fig. 3). Pellet size can be varied from $40-500 \mathrm{mg}$. All the lobsters can be fed in 8.5 hours.

In the new concept the main function is to control that the correct number of boxes are fed in every ring where feeding is ordered to do so. The program is written in Basic. The flow diagram in Fig. 4 shows the principle of the program. The timing of each feeding sequence is shown in Fig. 5.

At the moment a plant is being designed that should be able to produce 1000000 juveniles per year. In such a set up it would be desirable for the system to recognize different sections of the plant containing different lobster sizes and automatically adapt pellet size. In such a concept there would be 3-4 size groups spread over 10-20 sections.

\section{Other automation and instrumentation tasks}

Apart from feeding there are several other steps in the production process where automatic control would be desirable.

Brine shrimp are produced in a flow-through system. Water flow and feeding are regulated manually in accordance with water quality, and density and size of the brine shrimp. Automation would involve registration of water quality $\left(\mathrm{NH}_{4}^{+}, \mathrm{NO}_{2}^{-}\right.$, 


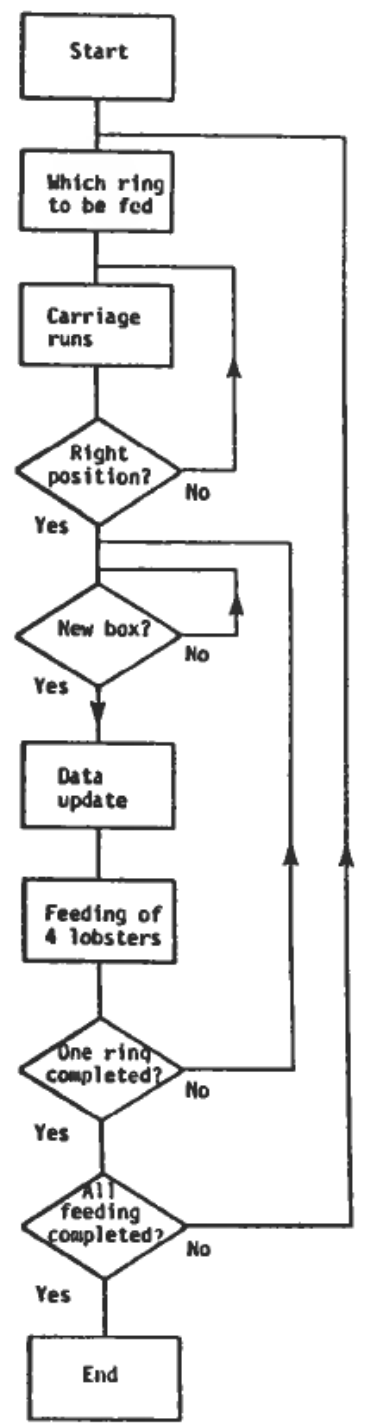

Figure 4. Flow diagram.

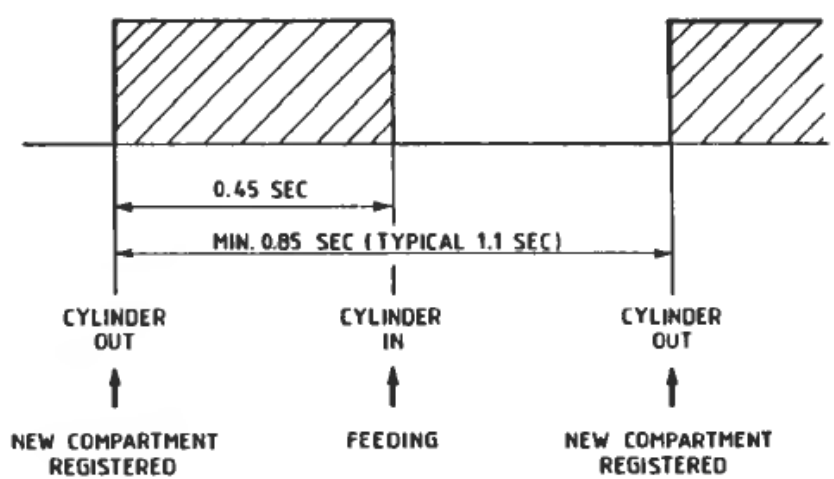

Figure 5. Timing diagram. 
$\mathrm{pH}, \mathrm{PO}_{2}$ ), temperature and brine density and control of water flow and food concentration.

Transfer of postlarvae from the tanks in the hatchery to the one lobster-one box system is a very laborious job. The process includes the following steps: (1) removal of postlarvae from tanks, (2) separation of individuals, (3) identification of healthy individuals, (4) release of selected individuals in the box system. Steps 1, 2 and 4 can probably be automated but step 3 would need the intervention of a human operator.

The use of cooling water from smelting works is not without problems; changes in the production can lead to rapid alterations in the temperature of the cooling water. Lobsters will tolerate such changes of $3-5^{\circ} \mathrm{C}$ in one hour, but ideally a temperature of $20-21^{\circ} \mathrm{C}$ should be maintained continuously, to produce maximum growth and reduce stress. Automatic control of pumps for regulating warm and cold water is, in fact, a necessity.

Further tasks in automation include control of water quality, detection of leakage in the large basin, back wash of filters and fault detection. A possible set up is illustrated in Fig. 6.

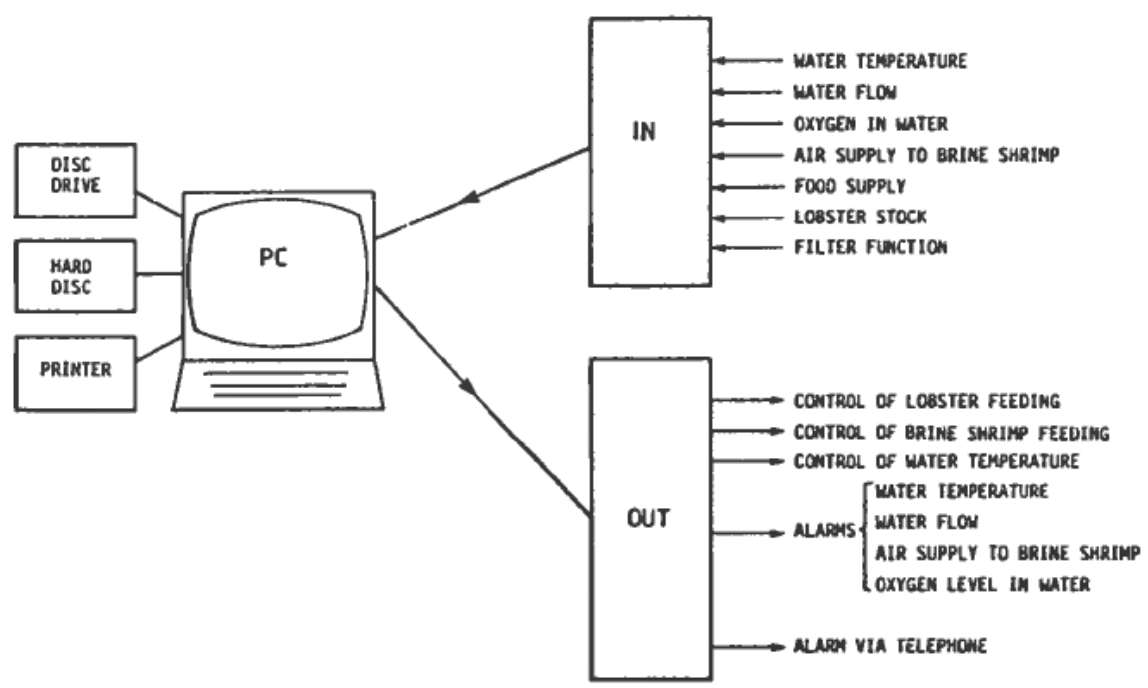

Figure 6. Proposed set up of instrumentation.

\section{ACKNOWLEDGMENTS}

We would like to thank Ola Buvik, Dag Hammer and Sigurd Lone for their contributions in developing the feeding equipment and Tiedemanns for permission to publish this paper.

\section{REFERENCES}

Beard, T. W., Richards, P. R., and Wickins, J. F. (1985). The techniques and practicability of year round production of lobsters, Hommarus gammarus $(L)$, in laboratory recirculation systems. MAFF Direct. Fish. Res., Fish. Res. Tech. Rep. 79, 22 pp.

Brisset, P., Versichele, D., Bossuyt, E., Ruyck, L. D., and Sorgeloos, P. (1982). High density flow through culturing of brine shrimp Artemia on inert feeds-preliminary results with a modified culture system. Aquacultural Engineering 1, 115-119. 
CARLSON, F. T. (1954). The American lobster fishery and possible applications of artificial propagation. Yale Conser. Stud. 3, 3-7.

Chang, E. S., and Conkuin, D. E. (1983). Lobster (Hommarus) hatchery techniques. In: J. P. McVey (Editor), CRC Handbook of Mariculture, Vol. I, Crustacean Aquaculture (CRC Press, Cleveland OH), pp. 271-275.

FAO, 1982, Yearbook of Fishery Statistics.

Van Olst, J. C., CarlBerg, J. M., and Hughes, J. T. (1980). Aquaculture. In: J. S. Cobb and B. F. Phillips (Editors), The Biology and Management of Lobsters, Vol. II (Academic Press, New York), Chapt. 10, pp. 333-384.

Wickins, J. F. (1983). On the track of profits from lobsters. Fish Farmer 6, 20-21. 